\title{
The emission lines radiated from accretion disk in cataclysmic variables
}

\author{
L. $\mathbf{H e}^{1,2}$, Y. H. Zhao ${ }^{2}$ and G. L. Lv ${ }^{1}$ \\ ${ }^{1}$ College of Physics Science and Technology, Xinjiang University, Xinjiang 830046, China \\ Email: helin@bao.ac.cn \\ ${ }^{2}$ National Astronomical Observatories, Chinese Academy of Sciences, Beijing 100012, China
}

\begin{abstract}
During the quiescent phases, the spectra of cataclysmic variables are studied. We model the fluxes by means of the photoionization code CLOUDY, and in the model it is assumed that the geometry of disks is cylindrical. Using this method, we input a series of reasonable parameters and get a spectral library.
\end{abstract}

Keywords. Cataclysmic variables, potoionization, accretion disk, emission lines

\section{Introduction}

A cataclysmic variable (CV) is a close binary system: a white dwarf (WD) with a low-mass, late-type star which fills its critical Roche surface and transfers matter to the primary. The accretion matter with the angular momentum can not settle immediately onto the WD but form a disk around it. Normally, the spectra of cataclysmic variables $(\mathrm{CVs})$ during their quiescent phases consist of the emission lines of HI, HeI, HeII, and CaII (Warner 1976). These emission lines from low ionization states of HI, HeI, HeII, FeII, CaII and OI showed by optical and IR spectra of most CVs (McClintock 1975). UV spectra of CVs radiate emission lines from more highly ionized regions are commonly observed: Ly $\alpha$, HeII, CII-CIV, NIII-NV, OI, OIII, OV, MgII, AlIII, SiII-IV (Jameson 1980; Fabian 1980 ). The Balmer emission lines are produced by the process of inner source radiating the outer regions of the disk (Williams 1980).

\section{Method}

Assuming that the geometry of disks which revolve around white dwarfs (WDs) are cylindrical, but in detail, disks are tapered because the outer parts of disks are thicker than the inner (warner 1999). With the low mass accretion rate, the spectrum radiated by the accretion disk model consists of two parts: the first part is a thermal continuum which is produced by the inner, hotter and optically thick portion of the disk, the second one is a series of emission lines originating from the cooler and outer part of the disk where the gas is optically thick in the lines and thin in the continuum. So during the quiescence phase of dwarf novae, Balmer lines emission can be explained by the emission from the optically thin outer regions of the disks (Williams 1980).

The photoionization code Cloudy (Ferland 2011) is used to model the emission-line fluxes. CLOUDY was born in the Institute of Astronomy, Cambridge, in August of 1978 and it is a large-scale spectral synthesis code which is designed for simulating the fully physical conditions within an astronomical plasma and then predict the emitted spectrum (Ferland 1998). The code can fix the ionization, temperature, and chemical abundance of a cloud and then predict its spectrum. With the minimum number of free parameters, all of this is done self-consistently (Ferland 2011) so it can simultaneously solve the equations 

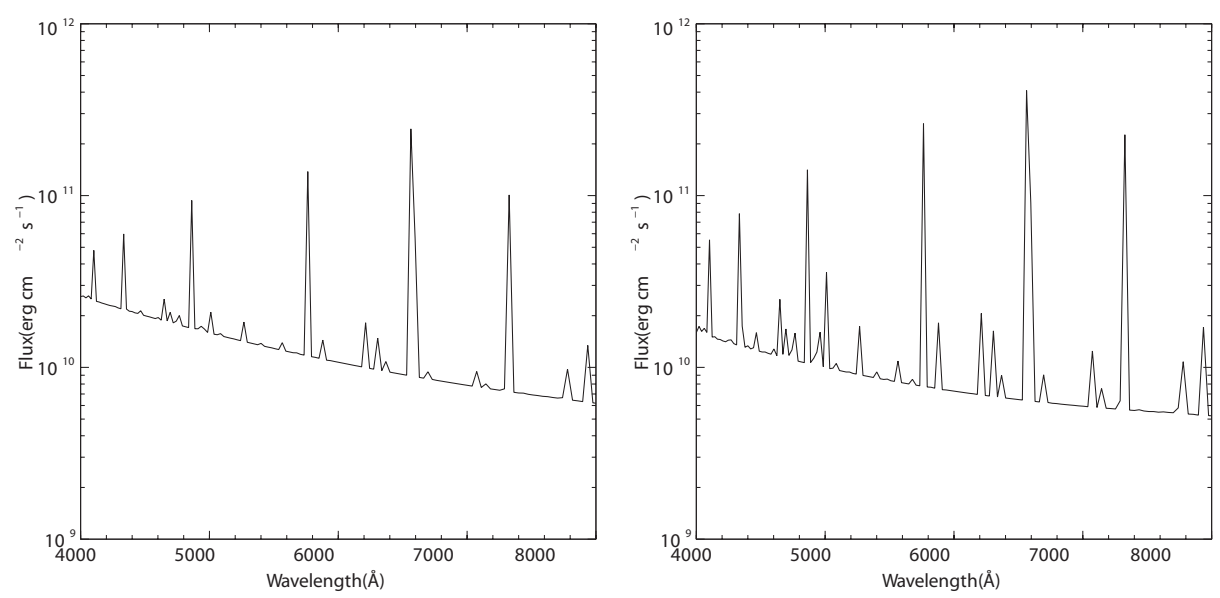

Figure 1. The spectra of two cataclysmic variables are the templets in the spectral library. The $\mathrm{x}$-axis and $\mathrm{y}$-axis are the wavelength in angstrom and flux $\left[\mathrm{ergcm}^{-2} \mathrm{~s}^{-1}\right]$, respectively. Most of the emission lines are hydrogen and helium recombination lines. The two spectra have different spectral energy distributions: the left one is a $28000 \mathrm{~K}$ blackbody and the right one is a $35000 \mathrm{~K}$ blackbody.

of thermal and statistical equilibrium for a cylinder of a dilute gas in the outer parts of disks that is being photoionized by an underlying thermal source (Moro-Martin 2001).

Some parameters are needed for the code: the temperature and luminosity of the inner parts of the disk and WD. These parameters describe the factors of the source. Then, the hydrogen density, radius and chemical composition of outer parts of the disk are also needed. These parameters describe the characteristics of the outer regions of the disk. It is also important to use other parameters to determine the covering factor and the geometry of the disk. In our model, we use the command "cylinder" to describe the disk. We give the reasonable ranges of these parameters and then we have created a spectral library (see Fig. 1).

\section{Discussion}

Now the spectral library is built, then these model spectra will be used to fit the observational spectra. Furthermore, it can be get the best fit parameters for CLOUDY which can describe the characters of the observational CVs. For example, we can try to class dwarf novaes using the differences of their chemical abundances.

\section{References}

Fabian, A. C., Pringle, J. E., Stickland, D. J. et al. 1980, MNRAS, 191, 457

Ferland, G. J., Korista, K. T., Verner, D. A. et al. 1998, PASP, 110, 761

Ferland, G. J. 2011, HAZY, a Brief Introduction to CLOUDY C10

Jameson, R. F., King, A. R., \& Sherrington, M. R. 1980, MNRAS, 191, 559

McClintock, J. E., Canizares, C. R., \& Tarter, C. B. 1975, AJ, 198, 641

Moro-Martin, A., Garnavich, P. M., \& Noriega-Crespo, A. 2001, AJ, 121, 1636

Warner, B. 1976, in IAU Symposium 73, Structure and Evolution of Close Binary Systems, ed. P. Eggleton, S. Mitton, and J. Whelan (Dordrecht: Reidel), p. 85.

Warner, B. 1999, ASP Conference Series, 157, 63.

Williams, R. E. 1980, ApJ, 235, 939. 